\title{
Efectos de un preparado probiótico en un modelo de colitis experimental crónica en ratones, inducida por la ingesta de dextrano sulfato sódico (DSS)
}

\section{Effects of a probiotic preparation in an experimental model of chronic colitis in mice, induced by the intake of dextranesulphate sodium (DSS)}

\author{
Bolívar-González $\mathrm{S}^{1}$, Talero Barrientos $\mathrm{E}^{2}$, Motilva Sánchez V² \\ ${ }^{1}$ Prog. Farmacia, Fac. Farmacia - Universidad del Atlántico (Baq, Col), ${ }^{2}$ Dep. de Farmacología, Fac. de Farmacia, Universidad de Sevilla (Sev, Esp)
}

\begin{abstract}
Resumen - La Colitis Ulcerosa es considerada una enfermedad inflamatoria intestinal de etiología desconocida. La ingesta de probióticos, representa una opción terapéutica novedosa para alterar la flora intestinal mediante el aumento de la concentración de bacterias beneficiosas y la reducción en los niveles de microorganismos patógenos.

Objetivo: Determinarlos efectos profilácticos o curativos de un preparado probiótico en un modelo de colitis experimental en ratones, inducida por el dextrano sulfato de sodio.

Materiales y Métodos: Los ratones son expuestos a 5 ciclos de tratamiento, cada ciclo consiste en la administración de dextrano sulfato de sodio durante una semana en el agua de bebida, al 0,7\%, seguida de agua potable durante 10 días. El preparado probiótico fue administrado antes de la inducción de la colitis o de forma conjunta con su inducción. Después de cada período, los animales son sacrificados y se realizan estudios macroscópicos e histológicos, se calcula el índice de actividad de la enfermedad, así como la caracterización de biomarcadores inflamatorios.

Resultados: La administración del preparado probiótico atenuó el índice de actividad de la enfermedad y la inflamación del colon después de los 5 ciclos del dextrano sulfato de sodio, así mismo redujo las alteraciones histológicas. Además, disminuyó la expresión proteica y génica de marcadores inflamatorios en el tejido del colon durante el periodo evaluado.

Conclusión: La administración del preparado probiótico, previene o retrasa los signos externos de la colitis, así como los niveles proteicos y génicos de marcadores inflamatorios asociados a ella.
\end{abstract}

Palabras Claves: Probióticos, colitis, enfermedad inflamatoria intestinal, citoquinas (Fuente: DeCS).

Correspondencia: Samir Bolívar González, Facultad de Química y Farmacia, Universidad del Atlántico, Km 7 Via Puerto Colombia, Teléfono 3197227, BarranquillaColombia. samirbolivargonzalez@hotmail.com.

Citar: Bolívar-González S, Talero Barrientos E, Motilva Sánchez V. Efectos de un preparado probiótico en un modelo de colitis experimental crónica en ratones, inducida por la ingesta de dextrano sulfato sódico (DSS). Cienc e Innovación en Salud [Internet]. 2015 Jan 1;3(1):1-1. Available from: http://publicaciones.unisimonbolivar.edu.co:82/rdigital/ojs/index.php/innovacionsalud/article /view/239/234

Parte de este trabajo se encuentran soportado en la investigación titulada «Efectos de un preparado probiótico en un modelo de colitis experimental crónica en ratones, inducida por la ingesta de dextrano sulfato sódico (DSS)» tesis para obtener el título de Máster en Atención Hospitalaria de la Universidad de Sevilla (España).

Recibido: Oct. 12 de 2014 / Modificado: Nov. 11 de 2014 / Aceptado: Nov. 21 de 2014.
Abstract - The ulcerous colitis is considered an inflammatory intestinal disease of unknown etiology. The intake of probiotic represents a new therapeutic option to alter the gut flora through the increase of concentrations of beneficial bacteria and the decrease of the levels of pathogenic microorganisms.

Objective: Determine prophylactic or curative effects of a probiotic preparation on a model of experimental colitis in mice induced by dextran sodium sulfate.

Materials and Methods: The mice were exposed to 5 treatment cycles, each consisting in administering dextranesulphate sodium in soda water $0.7 \%$ for one week, and then potable water for 10 days. The probiotic preparation was administered before inducing colitis or jointly with induction. After each period, the animals were sacrificed and macroscopic and histological studies, and the index of disease activity was calculated, as well as the characterization of inflammatory biomarkers.

Results: The administration of the probiotic preparation alleviated the index of disease activity and the colon inflammation after the 5 cycles of dextranesulphate sodium, reducing as well the histological alterations. Also, the protein and gene expression of the inflammatory markers in the colon tissue decreased during the evaluated period. Conclusion: The administration of the probiotic preparation prevents or retards the external signs of colitis, and protein and gene levels of inflammatory markers associated with it.

Keywords: Probiotics, colitis, intestinal inflammatory disease, cytokines (Source: MeSH, NLM).

\section{INTRODUCCIÓN}

La enfermedad inflamatoria intestinal (EII) es un grupo de enfermedades caracterizadas por la inflamación del intestino delgado y grueso e incluye principalmente Colitis Ulcerativa (CU) y la Enfermedad de Crohn (EC). Aunque la etiología de la Ell no se entiende completamente, se cree que es el resultado de la interacción de factores genéticos, inmunológicos, y los factores ambientales, incluyendo la microbiota intestinal (1).Ambas formas de Ell, se caracterizan por ser crónicas, deterioran significativamente la calidad de vida, requieren prolongadas intervenciones médicas y 
quirúrgicas, y representan una carga importante para la sociedad en general. Lo que hace particularmente difícil el tratamiento de esta enfermedad son sus causas, aún desconocidas. En la actualidad se viene estructurando la idea de que no se está ante una enfermedad en singular (EII), ni tampoco ante la simple dicotomía de una pareja de enfermedades "primas hermanas" (EC y la CU), sino ante un amplio espectro de procesos patológicos que surgen desde factores etiológicos y caminos patogénicos diferentes, para terminar en un hecho biológico con un común denominador, que es la inflamación crónica y recurrente de la pared intestinal (2). Durante años muchas teorías se han propuesto para explicar su patogénesis, incluyendo causas que van desde infecciosas a psicosomáticas, sociales, metabólicas, vasculares, genéticas, alérgicas, autoinmunes e inmunitarias $(3,4)$. En este sentido, se considera que estas enfermedades son la consecuencia de una respuesta inmune inadecuada que provocaría una inflamación mantenida en la mucosa intestinal y que ocurre en individuos susceptibles genéticamente como consecuencia de una compleja interacción entre factores medio-ambientales, microbiológicos e inmunes (5).

En el intestino normal, los macrófagos están condicionados por el microambiente de la mucosa para expresar un fenotipo no inflamatorio, traducido por una expresión disminuida de los receptores de la inmunidad innata y la limitada producción de citocinas proinflamatorias (6). La creciente evidencia apoya el papel de diversas citocinas liberadas por las células epiteliales e inmunes, en la patogénesis de la CU asociada a neoplasia. Se ha sugerido que existen dos mediadores claves en el proceso inflamatorio, la ciclooxigenasa-2 (COX-2), y sus productos, y el factor nuclear kappa $\beta$ (NF$\kappa \beta)$, y las moléculas producidas tras sus efectos transcriptores, los cuales serían los vínculos más destacados entre la inflamación y el cáncer. Recientemente, se ha demostrado que otros factores tales como el factor de necrosis tumoral alfa (TNF- $\alpha$ ) y la señalización de interleucina 6 (IL-6) promueven el crecimiento tumoral en modelos experimentales de cáncer asociado a colitis (CAC) (7).Además, se ha informado de que el aumento de la proliferación celular epitelial asociada con ciclos repetitivos de inflamación, daño, y la regeneración en caso de UC podría conducir a la carcinogénesis en el largo plazo. En este contexto, la $\mathrm{CU}$ puede ser modelada en ratones mediante la exposición repetida al sodio sulfato de dextrano (DSS), un agente con efectos tóxicos directos sobre el epitelio del colon. Este modelo ha demostrado las características clínicas e histopatológicas similares a los observados en
UC humana (8).En diversos modelos animales han descubierto un papel central de la flora intestinal en el desarrollo de la colitis, principalmente por la inducción de las células Th17 o células T reguladoras (9). La convergencia de los resultados demuestra que la flora intestinal desempeña un papel central en la patogénesis de esta enfermedad, es así, como los investigadores vienen realizando estudios clínicos que pretenden encontrar beneficios terapéuticos luego de la manipulación en la flora intestinal. Por lo anterior, cada día se reconoce un creciente interés en la comunidad científica sobre el papel protector de los probióticos en las enfermedades intestinales, especialmente en la Ell $(10,11)$.

Los probióticos son microorganismos vivos, que cuando llegan en cantidades suficientes al intestino en un estado activo, ejercen efectos positivos para la salud (12). En su mayoría incluyen bacterias y levaduras productoras de ácido láctico que alcanzan el intestino sin alteraciones, y además sin proporcionar daño al huésped $(13,14)$.En este sentido, por sus acciones inmunomoduladoras y antiinflamatorias, se conocen diferentes aplicaciones de los probióticos en la prevención de infecciones intestinales, cáncer y enfermedades cardiovasculares $(15,16)$.En el intestino, los probióticos son capaces de ocupar nichos de la mucosa, impidiendo a los patógenos invadir esas zonas. Además, ejercen una acción local semejante a los antibióticos contra microorganismos patógenos y disminuyen la producción de citocinas pro-inflamatorias, tales como interferón-gama (IFN- - ), TNF- $\alpha$ e interleucina 2 (IL-2). También presentan la capacidad de modular las vías dependientes del NF-k $\beta$, lo cual parece estar relacionado con el establecimiento de la tolerancia inmunológica (17). Sin embargo, pocas investigaciones han abordado el efecto de los probióticos en modelos experimentales de colitis, y los resultados que existen son contradictorios. En esta línea, se ha demostrado que la administración profiláctica del preparado probiótico redujo la inflamación intestinal y carcinogénesis (18); por el contrario, la administración de probióticos no altero el ambiente inflamatorio del colon en un modelo animal de colitis asociado a cáncer (10). Este estudio tuvo como objetivo determinar los efectos de un preparado probiótico, cuando se administra como tratamiento preventivo o concurrente con la colitis inducida por el DSS, en ratones. Se ha observado, en la secuencia de la inflamación crónica intestinal inducida en los ratones, los efectos del preparado probiótico sobre la expresión de COX-2, así como los niveles de citoquinas proinflamatorias, tales como TNF- $\alpha$. Los resultados 
demuestran que el preparado probiótico mejora la colitis crónica en ratones y brinda un potencial uso terapéutico en pacientes con Ell crónica.

\section{MATERIALES y MÉTODOS}

\section{ANIMALES DE EXPERIMENTACIÓN}

Los experimentos siguieron un protocolo estipulado por el Comité de Ética Animal de la Universidad de Sevilla, además, todos los experimentos estaban en conformidad con las recomendaciones de la Unión Europea en relación con la experimentación animal (Directiva del Consejo Europeo 2010/63/UE). Los animales fueron mantenidos en condiciones estándares de estabulización a 24-25 ํㅡ, doce horas de luz al día y alimentación controlada. En los estudios experimentales el número de animales utilizado por cada grupo ha estado comprendido entre 6-10. Se utilizaron ratones hembra de la cepa C57BL/6 de 6 semanas de edad, de peso comprendido entre $20-25 \mathrm{~g}$, suministrados por el Centro de Producción y Experimentación Animal de la Universidad de Sevilla.

\section{Preparado Probiótico}

El preparado probiótico utilizado se obtuvo de Actial Farmacéutica Lda(Portugal), es una mezcla de 5 cepas bacterianas productoras de ácido láctico (Lactobacillus acidophilus, Lactobacillus paracasei, Lactobacillus plantarum, Bifidobacterium longum, Bifidobacterium infantis).La dosificación empleada atendiendo a la bibliografía consultada, mencionan que por cada 100 gramos de ratón $/ 5$ billones de Unidades Formadoras de Colonias (UFC) de probiótico/día.

Anteriormente, fue necesario llevar a cabo experimentos in vitro para determinar el número de bacterias que sobreviven en presencia de DSS, y así comprobar que la viabilidad del probiótico no se ve influida por el DSS.

\section{Diseño de los Grupos EXPerimentales de ANIMALES}

1. Para el presente estudio se establecieron los siguientes grupos experimentales de animales:

1. Agua, constituido por 10 ratones que recibieron agua potable a lo largo de todo el experimento.

2. Probiótico, constituido por 10 ratones que recibieron el agua potable complementada con el preparado probiótico a lo largo de todo el experimento.

3. DSS, constituido por 15 ratones que recibieron 5 ciclos de DSS. Cada ciclo consistió en la administración oral cíclica de DSS al $0.7 \%$ durante 7 días consecutivos, seguido por un periodo de agua de 10 días.

4. DSS + Pre-Probiótico", constituido por 15 ratones que fueron pre-tratados con el preparado probiótico durante 14 días previos a la inducción de la colitis. Tras ese periodo, recibieron 5 ciclos de DSS al $0.7 \%$ y se continuó con la administración del preparado probiótico a lo largo de todo el experimento.

5. DSS + Probiótico, constituido por 15 ratones que recibieron 5 ciclos de DSS al $0.7 \%$, así como también el preparado probiótico a lo largo de todo el experimento.

\section{Modelo de Colitis Ulcerosa Crónica}

El modelo experimental utilizado es un modelo de inflamación intestinal crónica propuesto por Yeo y cols., (2006) (19). La colitis fue inducida por administración oral cíclica de DSS al $0.7 \%$ durante 7 días consecutivos, seguido por un periodo de agua de 10 días. El DSS provoca infiltración leucocitaria en la mucosa, lesiones inflamatorias y diarrea sanguinolenta. Los animales fueron sacrificados después de 5 ciclos ( 85 días) de DSS.

EVAluación de la SeVeridad de la Colitis y ANálisis MACROSCÓPICO DE LAS LESIONES

Durante todo el periodo experimental se valoraron las manifestaciones clínicas de la colitis para determinar el índice de actividad de la enfermedad (DAI), el cual engloba la presencia de diarrea, el sangrado rectal y la pérdida de peso de los animales. Estos tres parámetros se evaluaron por separado en una escala de cero (0) a tres (3) según el método propuesto por Gommeaux y cols., (2007) (20) (Tabla 1) y el promedio de los tres valores constituyó el DAl. Al finalizar el periodo experimental (5 ciclos de DSS), los animales fueron sacrificados mediante inyección i.p. de hidrato de cloral al $4 \%$ y se extirpó el colon en su totalidad para su análisis macroscópico, histológico y bioquímico. La relación peso/longitud del colon fue igualmente evaluada.

Tabla 1 Escala propuesta por Gommeaux y cols., (2007) para la determinación del índice de la actividad de la enfermedad (DAI).

\begin{tabular}{llcl}
\hline \hline Escala & Sangrado & $\begin{array}{l}\text { Pérdida } \\
\text { de peso } \\
\text { (\%) }\end{array}$ & $\begin{array}{l}\text { Consistencia de } \\
\text { heces }\end{array}$ \\
\hline 0 & Ninguno & $<1$ & $\begin{array}{l}\text { Deposiciones } \\
\text { normales }\end{array}$ \\
1 & $\begin{array}{l}\text { Pequeñas manchas de } \\
\text { sangre en heces; región anal } \\
\text { sin sangre. }\end{array}$ & $1-4.99$ & $\begin{array}{l}\text { Heces blandas no } \\
\text { adheridas al ano. }\end{array}$ \\
\hline \hline
\end{tabular}




\begin{tabular}{llcl}
\hline \hline Escala & Sangrado & $\begin{array}{l}\text { Pérdida } \\
\text { de peso } \\
\text { (\%) }\end{array}$ & $\begin{array}{l}\text { Consistencia de } \\
\text { heces }\end{array}$ \\
\hline 2 & $\begin{array}{l}\text { Grandes manchas de sangre } \\
\text { en heces; sangre en orificio } \\
\text { anal. }\end{array}$ & $5-10$ & $\begin{array}{l}\text { Heces muy } \\
\text { blandas } \\
\text { adheridas al ano. }\end{array}$ \\
\hline $\begin{array}{l}\text { Heces totalmente } \\
\text { hemorrágicas; sangre } \\
\text { extendida por todo el ano. }\end{array}$ & $>10$ & Diarrea intensa. \\
\hline \hline
\end{tabular}

\section{ESTUDIO HISTOLÓGICO DE LAS LESIONES DEL COLON}

Para llevar a cabo el análisis microscópico del daño colónico, se seleccionaron al azar 5 animales por grupo. Se procedieron a extraercon escalpelo secciones de zonas parcialmente ulceradas del colon de los ratones, que se sumergieron en formol al $4 \%$ durante 24 horas. A continuación, las muestras se sometieron a una deshidratación mediante alcoholes de concentración creciente y se incluyeron en parafina. Secciones de $5 \mu \mathrm{m}$ de grosor cortadas en un ultramicrotomo Leica Ultracut se sometieron a un proceso de desparafinación e hidratación para ser teñidas con hematoxilina/eosina. Dichas preparaciones fueron examinadas microscópicamente, utilizándose los objetivos $4 x, 10 x$ y 20x. La puntuación para la inflamación es una adaptación de Okayasu et al. (1990) (21): Grado 0: La cripta intacta, Grado 1: La infiltración de neutrófilos y la pérdida de la parte basal de la cripta, Grado 2: La infiltración de neutrófilos, con la pérdida de toda la cripta pero epitelio superficial intacto, Grado 3: La infiltración de neutrófilos con la pérdida tanto de la cripta como del epitelio superficial. El análisis de las imágenes fue realizado utilizando una cámara ALTRA 20 (Olympus CX41) acoplada a un sistema de análisis de imágenes automático (AnalySISgetIT).

\section{DETERMINACIÓN DE LA PRODUCCIÓN DE CITOCINAS}

Los niveles de la citocina pro-inflamatoria TNF- $\alpha$ fueron determinados por una técnica de inmunoanálisis enzimático cuantitativo tipo sandwich, utilizando el kit MurineTNF- $\alpha$ ELISA (Peprotech, GB).Para la realización de la técnica, las muestras fueron homogeneizadas en tampón fosfato sódico (PBS) ( $\mathrm{pH}$ 7.2) conteniendo albúmina bovina (BSA) $1 \%$ y centrifugadas $(12,000$ rpm, $10 \mathrm{~min}$ ). Los diferentes patrones $\mathrm{y}$ las muestras se pipetearon en pocillos recubiertos de un anticuerpo monoclonal específico para la citocina en estudio. Tras el lavado, se adicionó un anticuerpo policlonal específico para la citocina unido a una peroxidasa y los substratos, peróxido de hidrógeno y $\operatorname{TMB}\left(3,33^{\prime}, 5,5^{\prime}\right.$ tetrametilbencidina). La reacción enzimática produjo un color azul que cambió a amarillo tras la adición de ácido sulfúrico 1M. La intensidad de color se midió a $450 \mathrm{~nm}$ en un lector de placa. Los resultados fueron expresados en picogramos de citocina/mg de tejido.

\section{ESTUDIO DE LA EXPRESIÓN PROTEICA MEDIANTE WESTERN BLOT}

En muestras de mucosa colónica se valoraron los niveles de expresión de la proteína COX-2 mediante electroforesis en geles de dodecil sulfato sódico (SDS)/poliacrilamida y posterior transferencia a membrana de nitrocelulosa, y análisis inmunológico, usando anticuerpos monoclonales específicos (Western Blot). Las muestras fueron procesadas por homogenización en frío con tampón de lisis compuesto por Tris $\mathrm{HCl} 50 \mathrm{mM}$ pH 7.5, $\mathrm{MgCl} 28 \mathrm{mM}$, ácido etilen glicol-bis ( $\beta$-aminoetiléter) tetraacético (EGTA) $5 \mathrm{mM}$, EDTA $0.5 \mathrm{mM}$, leupeptina $0.01 \mathrm{mg} / \mathrm{ml}$, pepstatina 0.01 $\mathrm{mg} / \mathrm{ml}$, aprotinina $0.01 \mathrm{mg} / \mathrm{ml}$, fenilmetilsulfonil fluoruro (PMSF) 1mM y NaCl 250 mM. A continuación los homogeneizados fueron centrifugados $(12,000 \mathrm{rpm}, 15$ $\min , 4 ㅇ$ C) y posteriormente se recogió el sobrenadante, que se conservó a $-80^{\circ} \mathrm{C}$. La concentración de proteínas del homogeneizado fue determinada aplicando el método colorimétrico de Bradford (1976) (22).

La separación de las proteínas se llevó a cabo empleando geles de SDS-poliacril-amida al 10-12\%. Se cargaron $50 \mu \mathrm{g}$ de proteínas diluidas en el reactivo de Laemmly y $\beta$-mercaptoetanol, desnaturalizadas previamente a $100^{\circ} \mathrm{C}$. Una vez finalizada la electroforesis, las proteínas se transfirieron a una membrana de nitrocelulosa y posteriormente se bloquearon con leche desnatada en polvo ( $5 \% \mathrm{p} / \mathrm{v})$. Las membranas fueron incubadas con el anticuerpo primario específico para COX-2 durante toda la noche a 4 으. Al día siguiente, tras sucesivos lavados, se procedió a la incubación con el anticuerpo secundario específico y finalmente, al revelado de los complejos antígenoanticuerpo mediante un kit de quimioluminiscencia (SuperSignal ${ }^{\circledR}$ West FemptoChemiluminescentSubstrate) suministrado por Pierce (USA).

RT-PCR (ReAcción en Cadena de la Polimerasa en TRANSCRIPCIÓN INVERSA)

En muestras de colon se valoraron los niveles de expresión del ARNm del gen de TNF- $\alpha$. El ARN total fue extraído a partir de muestras de colon utilizando el kit comercial denominado SV Total RNA IsolationSystem (Promega, EE.UU.) en un ambiente libre de RNasa. La transcripción inversa se llevó a cabo utilizando1-2 mg de ARN con el SuperScript ${ }^{\mathrm{TM}}$ First-StrandSynthesisSystemfor RT-PCR (Invitrogen, EE.UU.), oligo (dT) primer 16, dNTP (0,5 mM) y un inhibidor de RNasa. Después de la incubación a $65{ }^{\circ} \mathrm{C}$ durante 5 minutos y $42{ }^{\circ} \mathrm{C}$ durante 50 
min, se detuvo la reacción con un choque térmico a 70 ${ }^{\circ} \mathrm{C}$ durante $15 \mathrm{~min}$. La reacción en cadena de la polimerasa (PCR) se llevó a cabo con la enzima polimerasa SYBR ${ }^{\circledR}$ Premix Ex Taq ${ }^{\mathrm{TM}}$ (TaKaRaBiolnc, Shiga, Japón) y $0,2 \mu \mathrm{M}$ de cada conjunto de cebadores para TNF- $\alpha$. El cDNA fue amplificado por 35 ciclos de desnaturalización a $95{ }^{\circ} \mathrm{C}$ durante $3-5$ segundos, hibridación a $55-60{ }^{\circ} \mathrm{C}$ durante $10-20$ segundos, y extensión a $72{ }^{\circ} \mathrm{C}$ durante $6-15$ segundos. Las secuencias de los primer utilizados fueron los siguientes: $5^{\prime}$ GAT CTC AAA GAC AAC CAA CTA GTG 3' (hacia delante), 5' CTC CAG CTG GAA GAC TCC TCC CAG $3^{\prime}$ (al revés) para TNF- $\alpha$ y 5 '-GTG GGC CGC CCT AGG CAC AG-3 ' (hacia adelante), 5'-AGA GGA GGAGGA TGC GGC AG T-3 '(al revés) para GADPH como control de la PCR.

\section{TRATAMIENTO ESTAdístico dE LOS RESULTAdOS}

Los resultados han sido expresados como la media aritmética, error estándar, y la significación de las diferencias entre los grupos ha sido evaluada utilizando el análisis de la varianza (test de ANOVA) seguido del test de Tukey-Kramer para datos paramétricos y la $\mathrm{U}$ de Mann-Whitney para datos no paramétricos. El análisis estadístico ha sido realizado utilizando el programa informático GraphPadPrisma ${ }^{\circledR} 5.01$.

Las imágenes de histología y western blot son representativas de al menos tres experimentos realizados en diferentes días.

\section{RESULTADOS}

Efectos del Preparado Probiótico sobre la Actividad Clínica DE LA ENFERMEDAD Y LOS DAÑOS MACROSCÓPICOS EN EL COLON

La administración oral de 5 ciclos de DSS al 0,7\% en el agua de bebida indujo en los animales un cuadro importante de colitis inflamatoria crónica, que se correlacionó positivamente con el número de ciclos de DSS administrados. En el grupo control DSS, el análisis del DAI, referido a los signos externos de la colitis (presencia de diarrea, sangrado rectal y pérdida de peso) demostró que la enfermedad ya estaba presente a partir del segundo ciclo y su gravedad se incrementó gradual y significativamente a medida que los ciclos con DSS avanzaban. Sin embargo, en los grupos DSS + preprobiótico y DSS + probiótico se observó una disminución significativa en los valores del DAI a lo largo de todos los ciclos ensayados, con respecto al grupo control DSS (figura 1).

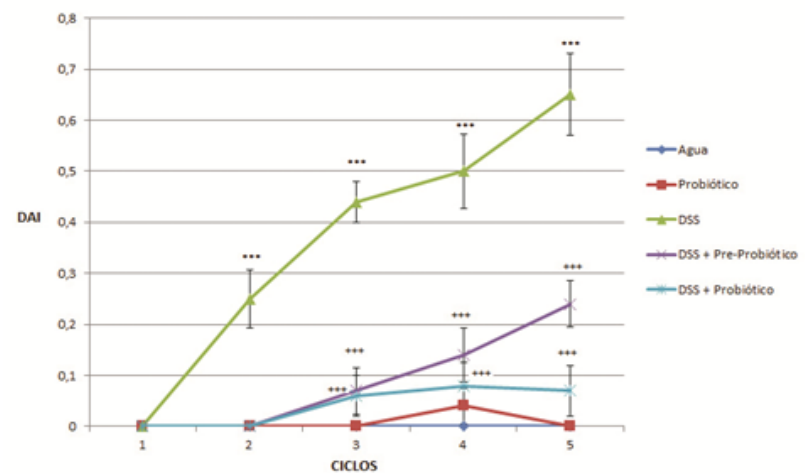

Figura 1 Índice de actividad de enfermedad (DAI) en el modelo experimental de colitis ulcerosa inducida por dextrano sulfato sódico (DSS) en ratones. El DAI se calcula como la media de las puntuaciones de sangrado, la pérdida de peso y la consistencia de las heces. $(* * *)$ $p<0.05$ vs. Agua, $(+++) p<0.05$ vs. DSS.

Una vez que los diferentes grupos de animales fueron sacrificados, medimos la longitud del tejido colónico, aplicado como un indicador de inflamación. Como era de esperar, los resultados evidenciaron un marcado acortamiento del colon en los animales que únicamente recibieron DSS. Sin embargo, en los grupos DSS + preprobiótico y DSS + probiótico se detectó una mayor longitud del colon, lo cual puede ser indicativo de cierta mejoría en dichos animales tras la administración del preparado probiótico (figura 2 y 3 ).

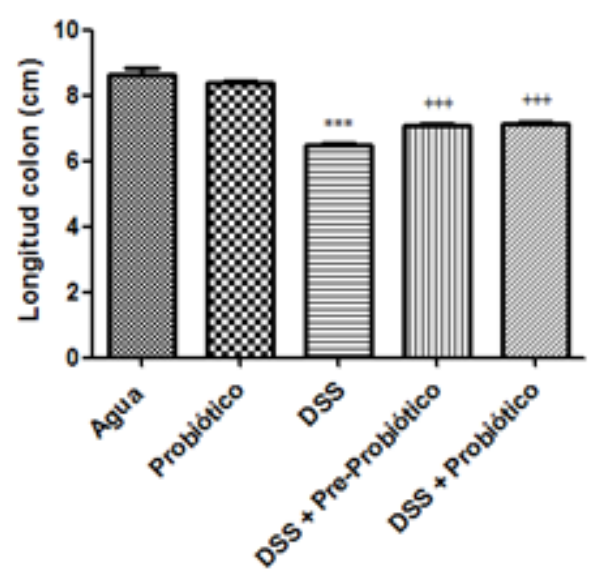

Figura 2 Proporción de longitud de tejido colónico de los grupos en el estudio. $\left(^{* * *}\right)$ p $<0.001$ vs. Agua, (+++) $p<0.001$ vs. DSS. 


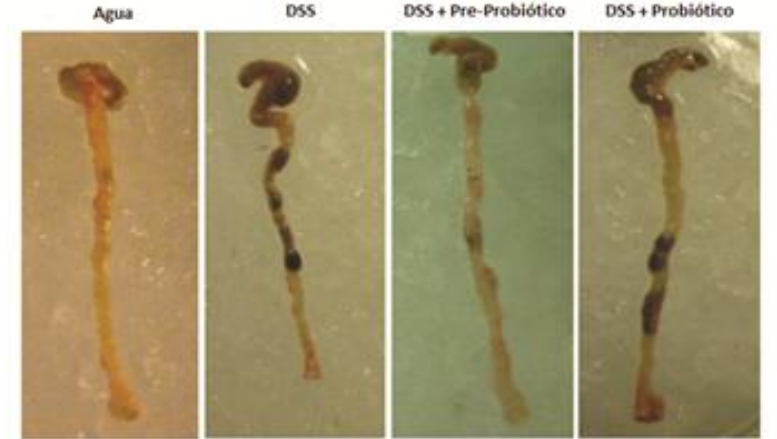

Figura 3 Imágenes representativas del aspecto macroscópico de la mucosa colónica de animales de los diferentes grupos del estudio: Agua, DSS, DSS + Pre-Probiótico y Probiótico.

EFECTOS DEL PREPARADO PROBIÓTICO SOBRE LOS DAÑOS MICROSCÓPICOS EN EL COLON

En los cortes histológicos de tejido colónico de animales sanos de nuestro estudio (figura 4A) se observó una estructura normal sin inflamación, displasia o ulceración. Sin embargo, la administración de 5 ciclos de DSS al $0,7 \%$ en el agua de bebida provocó una inflamación generalizada, caracterizada por abscesos en las criptas y alteración de la estructura glandular. Además, se detectó la presencia de un infiltrado inflamatorio leucocitario con granulocitos, monocitos y macrófagos en la mucosa y submucosa. Sin embargo, no se observó destrucción total del epitelio superficial (figura 4B). En los cortes histológicos obtenidos en los gruposDSS + pre-probiótico y DSS + probióticose apreció una notable disminución del infiltrado inflamatorio y una mayor conservación de la estructura glandular con respecto al colon del grupo control DSS (figura 4C-D).

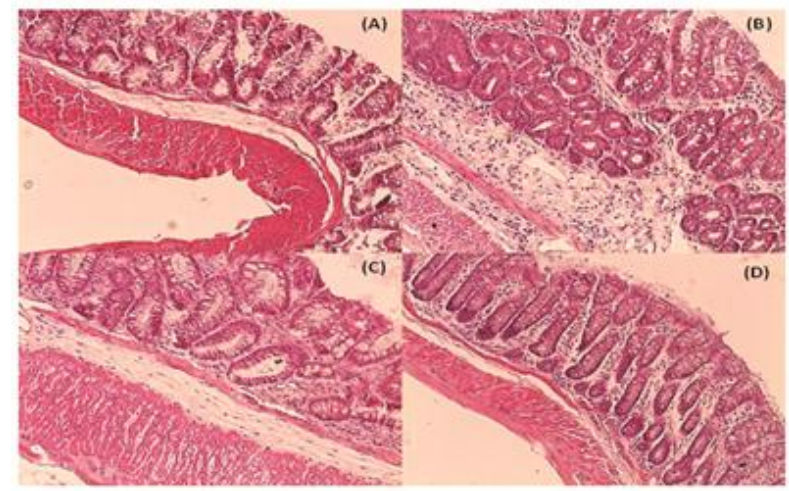

Figura 4 Imágenes microscópicas del colon de los grupos en estudio. (A) Morfología normal de la mucosa del colon en animales sanos. (B) Estructura de mucosa afectada en animales del grupo control de dextrano sulfato de sodio (DSS), en los cuales son evidentes la inflamación y displasia leve. (C-D) Estructura de la mucosa del colon de animales de grupos DSS + Pre-Probiótico y DSS + Probiótico, respectivamente, en los cuales se observa una clara disminución del infiltrado de células inflamatorias, así como también una mayor conservación de la estructura de las criptas. Tinción con hematoxilina y eosina (10X).
En los ratones a los cuales se les administraron los 5 ciclos de DSS (Fig. 4B-C y D) (Tabla 2), se observó la puntuaciónmás alta de inflamación, según la escala de Okayasu et al. (21). Sin embargo, el daño microscópico del colon fue significativamente menor en todas las regiones del colon en los animales de los grupos DSS + pre-probiótico y DSS + probiótico en el período estudiado, en comparación con el respectivo grupo control DSS $(P<0,05)$ (Fig. 4C-D). No hubo diferencias en los parámetros histológicos de colitis entre los grupos tratados con el preparado probiótico (Tabla 2).

Los datos relativos a la incidencia de displasia leve se presentan en la Tabla 2 y se expresan como el número de ratones portadores de lesiones, dividido por el número total de animales por grupo. La severidad de la displasia se correlacionó positivamente con el número de ciclos administrados DSS y el grado de inflamación. Después de 5 ciclos de DSS, los animales desarrollaron displasia de bajo grado, que se detectó en el $80 \%$ de los ratones del grupo control DSS. Se observó una reducción de la incidencia de displasia de bajo grado en ratones pre-tratados y tratados con el preparado probiótico en comparación con el grupo control de DSS (60\% y $40 \%$, respectivamente).

Tabla 2 Evaluación histológica e incidencia de displasia leve, según Okayasu et al. [21], en el modelo de colitis crónica inducida por dextrano sulfato de sodio (DSS).

\begin{tabular}{lccc}
\hline \hline Grupo & $\begin{array}{c}\text { No. de } \\
\text { animales }\end{array}$ & $\begin{array}{c}\text { Puntuación de } \\
\text { Colitis }\end{array}$ & $\begin{array}{c}\text { Grado de } \\
\text { displasia leve }\end{array}$ \\
\hline Agua & 10 & 0 & 0 \\
Probiótico & 10 & 0 & 0 \\
DSS & 10 & $1,92 \pm 0,1^{*}$ & $8 / 10(80 \%)$ \\
DSS + Pre-probiótico & 10 & $1,18 \pm 0,1^{\S}$ & $6 / 10(60 \%)$ \\
DSS + Probiótico & 10 & $1,23 \pm 0,2^{\S}$ & $4 / 10(40 \%)$ \\
\hline \hline$\left.{ }^{*}\right) p<0,001$ con respecto al grupo control Agua. (\$) $p<0,05$ con respecto al grupo control DSS.
\end{tabular}

EFECTOS DEL PREPARAdO PROBIÓtICO SOBRE LOS NIVELES DE EXPRESIÓN PROTEICA DE ENZIMAS Y CITOCINAS PRO-INFLAMATORIAS

Para apoyar los efectos anti-inflamatorios beneficiosos del preparado probiótico en la colitis ulcerosa e investigar su posible mecanismo de acción, se determinaron los niveles de expresión proteica de la enzima COX-2, así como la producción y niveles de expresión génica de la citocina pro-inflamatoria TNF- $\alpha$; las cuales han sidoaltamente correlacionado con la Ell (Fig. 5, 6 y 7). El nivel de expresión de la proteína COX-2 en el colon fue muy elevado en los ratones con colitis inducida por la administración de 5 ciclos de DSS al $0.7 \%$, respecto a los grupos control agua y control probiótico, en los cuales se detectaron niveles muy reducidos de dicha proteína. Interesantemente, los animales que recibieron DSS + pre-probiótico y DSS + probiótico 
presentaron unos niveles de expresión de esta enzima significativamente más bajos con respecto al grupo control DSS.

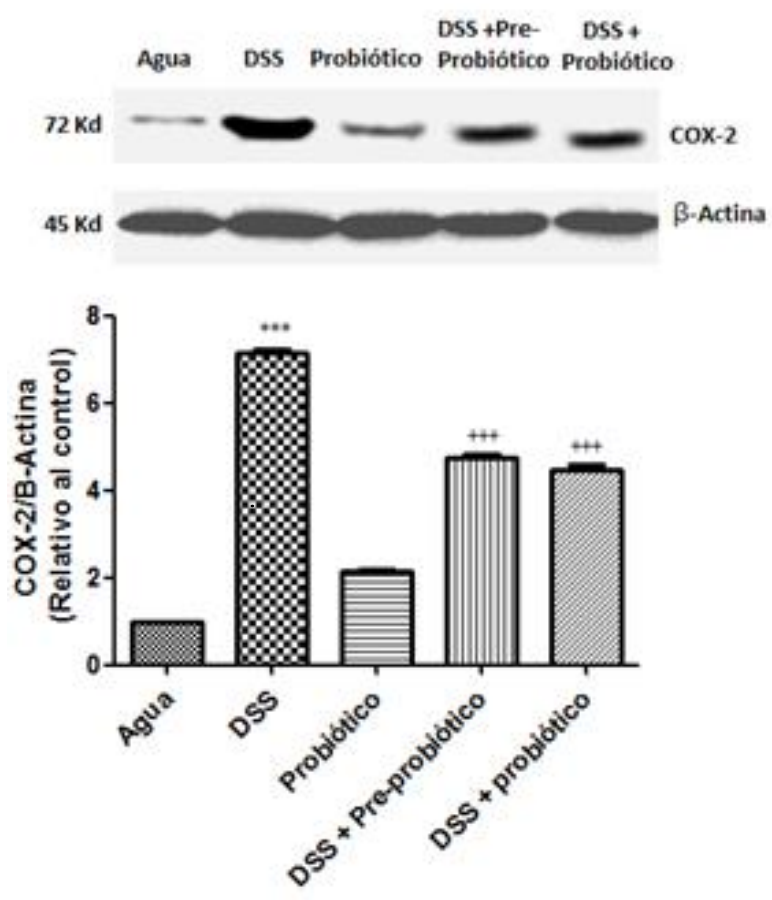

Figura 5 Análisis representativo mediante western blot de la proteína ciclooxigenasa-2 (COX-2) tras la administración del preparado probiótico en ratones con colitis inducida por 5 ciclos de dextrano sulfato sódico (DSS) al $0.7 \%$. El análisis densitométrico fue valorado comparándolo con el control de $\beta$-actina. $\left({ }^{* *}\right) p<0.001$ vs. Agua, $(+++)$ $p<0.001$ vs. DSS. La expresión de $\beta$-actina fue la misma en todas las muestras.

Los resultados correspondientes a la producción de TNF-ademostraron un incremento significativo de los niveles de este parámetro en el grupo control DSS, hasta llegar al valor de $1,453 \pm 0.25 \mathrm{pg} / \mathrm{mg}$ tejido en relación con los obtenidos en mucosa colónica de animales sanos que únicamente recibieron agua $(0,51 \pm 0.13 \mathrm{pg} / \mathrm{mg}$ tejido). Por el contrario, en los grupos DSS + preprobiótico y DSS + probiótico, los valores de esta citocina disminuyeron significativamente con respecto al grupo control DSS (figura 6).

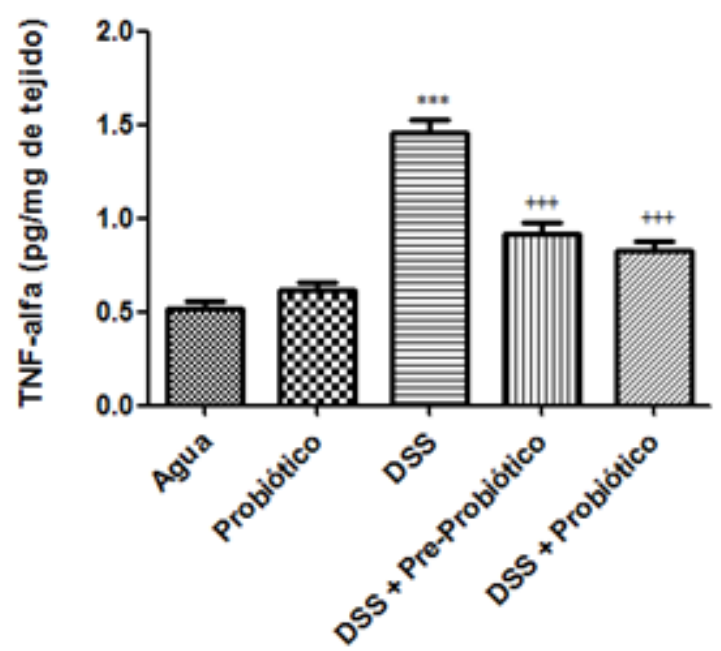

Figura 6 Producción de la citocina factor de necrosis tumoral alfa (TNF$\alpha, \mathrm{pg} / \mathrm{mg}$ tejido) en tejido de colon, siguiendo el modelo experimental de colitis crónica inducida por DSS al 0,7\% y la administración del preparado probiótico. $\left({ }^{* *}\right) \mathrm{p}<0.001$ vs. Agua $(++) \mathrm{p}<0.01$ vs. DSS.

En cuanto a la expresión génica del TNF-? en el colon, se encontró niveles muy elevados de esta citocina,en aquellos ratones con colitis inducida por la administración de los 5 ciclos de DSS al $0.7 \%$, respecto a los grupos control sano. Interesantemente, los animales de los grupos DSS + pre-probiótico y DSS + probiótico mostraron unos niveles de expresión de esta citocina significativamente más bajos con respecto al grupo control DSS (Figura 7).

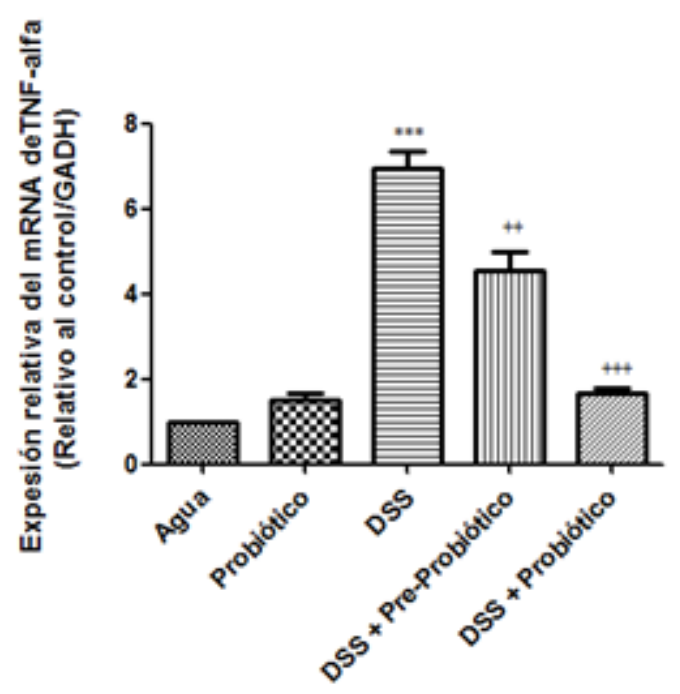

Figura 7 Expresión génica de la citocina factor de necrosis tumoral alfa (TNF-) en tejido de colon, siguiendo el modelo experimental de colitis crónica inducida por DSS al $0,7 \%$ y la administración del preparado probiótico. Los resultados se analizaron por el método de cuantificación relativa $(2-\Delta \Delta C T)$. 


\section{DISCUSIÓN}

Los probióticos son bacterias vivas que, cuando se administran en cantidad suficiente, presentan propiedades beneficiosas para la salud del individuo que los ingiere. Se ha demostrado que la flora intestinal desempeña un papel importante en la iniciación y el mantenimiento de la $\mathrm{CU}(23,24)$; es así, como diversos estudios han confirmado que la administración de bacterias probióticas confieren efectos beneficiosos en varios modelos de colitis experimental en animales (2530), y en ensayos clínicos con humanos(31, 32); principalmente los probióticos actúan regulandoel microambiente de la mucosa intestinal. Sin embargo, los mecanismos moleculares por los cuales estos probióticos ejercen sus efectos terapéuticos aún no han sido esclarecidos.

El objetivo principal de la investigación ha sido valorar los efectos de la administración, tanto de forma preventiva como terapéutica de un preparado probiótico en un modelo experimental de colitis crónica en ratones inducida por la administración cíclica de DSS al $0,7 \%$ en el agua de bebida. El tratamiento con DSS es un modelo bien aceptado para la Ell, ya que se considera que el proceso inflamatorio provocado por este tóxico reproduce en buena medida las manifestaciones clínicas, macroscópicas, histológicas e inmunológicas de la colitis humana clínica (33). En el estudio se ha llevado a cabo la evaluación de la respuesta inflamatoria mediante el análisis histológico de las lesiones colónicas, así como la cuantificación de biomarcadores asociados con la colitis, incluyendo la citocina TNF- $\alpha$ y la enzima inducible COX2.

Los resultados evidenciaron que la administración oral de 5 ciclos de DSS en el agua de bebida indujo en los animales un cuadro importante de colitis inflamatoria crónica, que se correlacionó positivamente con el número de ciclos de DSS administrados. El daño colónico fue confirmado por la reducción detectada en la longitud del colon en estos animales. El tratamiento con el probiótico, tanto de manera preventiva como terapéutica, atenuó los signos externos de la colitis, tales como la presencia de diarrea, el sangrado rectal y la pérdida de peso, así como también la reducciónen el acortamiento del colon observado en los animales que recibieron DSS. Estos resultados se relacionan con estudios anteriores, en los cuales demostraron que los probióticos suprimen la colitis en varios modelos experimentales en animales y en pacientes con Ell (34, 28, 35).

Aunque el mecanismo de la colitis experimental inducida por DSS no se comprende totalmente, la mayoría de los estudios in vivo sugieren que este tóxico causa colitis debido a los daños que produce directamente sobre las células epiteliales colónicas ubicadas en la base de las criptas. Como resultado se produce una respuesta inflamatoria en la mucosa, caracterizada por la activación de linfocitos Th1 y Th2 y la consecuente liberación de citocinas pro-inflamatorias $(36,37)$. En estas condiciones, estas moléculas son responsables de la infiltración de la mucosa y de la activación de diferentes poblaciones de leucocitos que contribuyen al desarrollo de la colitis (38). De manera similar, los resultados del estudio histológico mostraron que el colon de los animales que recibieron únicamentelos 5 ciclos de DSS presentaba una inflamación generalizada, detectándose la distorsión en la arquitectura de las criptas y la presencia de un infiltrado inflamatorio a nivel de la mucosa y de la submucosa. Además, la mayoría de los animales de este grupo desarrollaron bajo grado de displasia. Los datos microscópicos obtenidos en los animales que los grupos DSS + pre-probiótico y DSS + probiótico confirmaron los resultados macroscópicos, reflejando una atenuación de la desestructuración de la mucosa y una importante reducción del infiltrado inflamatorio, así como una menor incidencia de displasia leve. De acuerdo con nuestros resultados, Appleyard y cols (2011) (18) reportóque los animales tratados con el probiótico VSL\#3 presentaron un daño microscópico significativamente menor con el grupo control, en un modelo de colitis con trinitrobencenosulfónico (TNBS) asociado a displasia.

Considerando que la disminución en la infiltración de las células inflamatorias en la mucosa podría ser debida a una reducción en los niveles de ciertas citocinas proinflamatorias, el siguiente paso fue determinar la expresión de la citocina TNF- $\alpha$ en la mucosa colónica de los animales del estudio. Los resultados mostraron una disminución significativa en la producción de esta citocina así como su expresión génica, en los grupos de ratones tratados con DSS + pre-probiótico y DSS + probiótico, en comparación con el grupo control DSS. Estos datos concuerdan con estudios previos que han señalado la eficacia y efectos beneficiosos de cepas probióticas bacterianas, en la reducción de marcadores pro-inflamatorios. En este sentido, Madsen y cols., (2001) (39), demostró que elprobiótico VSL\#3fue capaz de disminuir la gravedad de la colitis en ratones knockout en el gen de la interleucina 10, debido en parte a la disminución de la producción de TNF- $\alpha$.

El hecho de que el preparado probiótico reduzca la inflamación asociada con la colitis producida por DSS, 
según el modelo experimental utilizado, es una característica muy interesante con respecto al potencial de estas bacterias como agentes terapéuticos. Sin embargo, estas propiedades beneficiosas observadas en el trabajo no coinciden con los resultados de un estudio previo realizado por Gaudier y cols., (2005) (40). Estos autores evidenciaron que el tratamiento con una mezcla de probióticos de manera curativa no fue capaz de revertir los daños macroscópicos e histológicos, ni el incremento en los niveles de TNF- $\alpha$ asociados a la administración de DSS. El hecho de que se hayan observado resultados contradictorios en ambos trabajos podría explicarse por las diferentes condiciones experimentales usadas. Así, Gaudier y cols., (2005) (40), llevaron a cabo un modelo de colitis moderada inducida por la administración de DSS al 1\% durante cinco días y mantenida por la adición de DSS al $0.2 \%$ durante 14 días. En dicho estudio, el preparado bacteriano fue administrado mediante sonda oral durante la etapa de mantenimiento de la colitis, es decir únicamente durante 2 semanas. Por el contrario, en nuestro el modelo experimental, la inflamación crónica ha sido inducida por la administración de diferentes ciclos de DSS (un total de 5 ciclos), y el preparado probióticoha tenido lugar durante un periodo de tiempo que ha comprendido aproximadamente 3 meses. Consideramos nuestro modelo especialmente interesante, ya que al alternar periodos de daño/remisión presenta una mayor semejanza con la colitis humana clínica.

En otra línea de trabajo, el interés se ha centrado en la relación de la enzima inducible COX-2 con el proceso inflamatorio crónico. Numerosos artículos demuestran cómo esta proteína aumenta en la Ell generando múltiples productos que agravan el proceso patológico $(41,42)$. Además, evidencias previas sugieren que esta enzima desempeña un importante papel en la etiopatogénesis del Cáncer Colorrectal (CCR). Este hecho ha sido confirmado en un estudio llevado a cabo por Taleroy cols., (8) utilizandoun modelo de inflamación intestinal crónica, provocada por la ingestión de DSS en el agua de bebida, que evoluciona a displasia y CCR (8). En dicho trabajo se evaluó la progresión de la enfermedad a lo largo del tiempo, sacrificando los animales luego de recibir 5, 10 ó 15 ciclos de DSS. Los resultados demostraron que los mayores niveles de expresión de la proteína COX-2 coincidieron con la máxima respuesta inflamatoria (que tuvo lugar en el 10응 ciclo) y posteriormente disminuyeron. Por ello, dichos resultados confirman el hecho de que esta enzima se induce en una fase temprana durante el proceso inflamatorio y está implicada en el inicio del cáncer.
Las primeras evidencias que muestran el efecto de los probióticos en la expresión de COX-2 han sido publicadas, por Otte y cols., (2009) (43). Estos autores observaron en células de cáncer de colon, una marcada disminución en la expresión de esta proteína, así como la secreción de prostaglandina 2 (PGE2) por acción de estas bacterias. En la misma línea, se ha demostrado que los probióticos podrían reducir la expresión de la COX-2 en un modelo de daño hepático inducido por una dieta alta en grasas en ratas jóvenes (44). Otro estudio reveló que el Lactobacillus casei, una de las cepas que componen nuestro preparado probiótico, atenuó la expresión de COX-2 en un modelo de colitis inducida por TNBS (45). En el modelo animal de colitis crónica utilizado se confirmó que la administración de DSS aumentaba significativamente los niveles de expresión de esta enzima inducible. Interesantemente, el tratamiento tanto preventivo como terapéutico del preparado probiótico del estudio provoca una marcada disminución en la expresión de la proteína COX-2.

Como se ha mencionado anteriormente, esta investigación se ha centrado en evaluar los efectos de la administración de un preparado probiótico, tanto de forma preventiva como terapéutica, en la colitis crónica. Por ello, un grupo de animales recibió este preparado durante dos semanas antes del inicio de la enfermedad y otro grupo justo en el momento de la inducción de la colitis. Los resultados confirmaron que en ambos grupos de animales, el preparado probiótico mostró efectos beneficiosos en la colitis inflamatoria; sin embargo no se apreciaron diferencias significativas entre los dos grupos de ratones. Por tanto, se concluye que en el modelo utilizado no hay diferencias entre los beneficios de la administración profiláctica del preparado probiótico frente a la preventiva.

En resumen, los resultados confirman que el preparado probiótico es eficaz contra la colitis crónica experimental inducida por DSS, ya que reduce el grado de inflamación, la incidencia de displasia leve y la infiltración de neutrófilos. Del mismo modo, también se ha observado una atenuación en la expresión y producción de biomarcadores asociados con la colitis, incluyendo la citocina TNF- $\alpha$ y la enzima COX-2, tras su administración crónica.

\section{AgradeCIMIENTOS}

El autor expresa su agradecimiento al Centro de Investigación, Tecnología e Innovación (CITIUS) de la Universidad de Sevilla por la prestación de asistencia técnica para la investigación. 


\section{REFERENCIAS BIBLIOGRÁFICAS}

1. Wasilewski A, Zielińska M, Storr M, Fichna J. Beneficial Effects of Probiotics, Prebiotics, Synbiotics, and Psychobiotics in Inflammatory Bowel Disease. Inflamm Bowel Dis. 2015; 0: 1-9. http://dx.doi.org/10.1097/MIB.0000000000000364

2. Sánchez-Fayos CP, Martín MJ, Porres JC.Multifactorial etiology and pathogenic factors in inflammatory bowel disease. GastroenterolHepatol. 2009; 32 (9):633-52. doi: 10.1016/j.gastrohep.2009.02.011.

3. Wen Z, Fiocchi C. Inflammatory bowel disease: autoimmune or immune-mediated pathogenesis? ClinDev Immunol. 2004; 11: 195-204. http://dx. doi.org/10.1080/17402520400004201.

4. Kaser A, Zeissing S, Blumberg RS. Inflammatory bowel disease. Annu Rev Immunol. 2010; 28: 573621. doi: 10.1146/annurev-immunol-030409101225.

5. Abraham C, Cho JH.Inflammatory Bowel Disease. N Engl J Med. 2009; 361(21): 2066-2078. doi: 10.1056/NEJMra0804647.

6. Elson CO, McCracken VJ, Dimmitt RA, Lorenz RG, Weaver CT. Experimental models of inflammatory bowel disease reveal innate, adaptive and regulatory mechanisms of host dialogue with the microbiota. Immunol Rev. 2005; 206: 260-76. doi: 10.1111/j.0105-2896.2005.00291.x

7. Kraus S, Arber N. Inflammation and colorectal cancer. Current Opinion in Pharmacology. 2009; 9: 405-10. doi: 10.1016/j.coph.2009.06.006.

8. Talero E, Sánchez-Fidalgo S, Villegas I, de la Lastra CA, Illanes $M$, Motilva $V$. Role of different inflammatory and tumor biomarkers in the development of ulcerative colitis-associated carcinogenesis. Inflamm Bowel Dis. 2011; 17: 696710. doi: $10.1002 /$ ibd.21420

9. Peloquin JM, Nguyen DD. The microbiota and inflammatory bowel disease: insights from animal models. Anaerobe. 2013; 24:102-6. doi: 10.1016/j.anaerobe.2013.04.006.

10. Arthur JC, Gharaibeh RZ, Uronis JM, et al.VSL\#3 probiotic modifies mucosal microbial composition but does not reduce colitis-associated colorectal cancer. Sci Rep. 2013; 3: 2868. doi: 10.1038/srep02868.

11. Nagpal R, Kumar A, Kumar M, et al.Probiotics, their health benefits and applications for developing healthier foods: a review. FEMS MicrobiolLett. 2012; 334: 1-15. doi: 10.1111/j.15746968.2012.02593.x.
12. Veerappan GR, Betteridge J, Young PE. Probiotics for the treatment of inflammatory bowel disease. Current Gastroenterology Reports. 2012; 14 (4): 324-333. doi: 10.1007/s11894-012-0265-5.

13. De Vrese $M$, Schrezenmeir J.Probiotics, prebiotics, and synbiotics. Advances in Biochemical Engineering/Biotechnology. 2008; 111: 1-66. doi: 10.1007/10_2008_097.

14. Looijer-van Langen MAC, Dieleman LA.Prebiotics in chronic intestinal inflammation. Inflammatory Bowel Diseases. 2009; 15 (3): 454-462. doi: 10.1002/ibd.20737.

15. E Isolauri, P Kirjavainen, and S Salminen. Probiotics: a role in the treatment of intestinal infection and inflammation? Gut. 2002; 50(Suppl 3): iii54-iii59. doi: 10.1136/gut.50.suppl_3.iii54.

16. M. G. Redman, E. J. Ward and R. S. Phillips.The efficacy and safety of probiotics in people with cancer: a systematic review. Ann Oncol. 2014; 25 (10): 1919-929. doi: 10.1093/annonc/mdu106.

17. Van Baarlen P, Troost FJ, van Hemert $S$, et al. Differential NF-kB pathways induction by Lactobacillus plantarum in the duodenum of healthy humans correlating with immune tolerance. Proc Nat AcadSci USA. 2009; 106, 23712376. doi: 10.1073/pnas.0809919106.

18. Appleyard CB, Cruz ML, Isidro AA, et al.Pretreatment with the probiotic VSL\#3 delays transition from inflammation to dysplasia in a rat model of colitis-associated cancer. Am J PhysiolGastrointest Liver Physiol. 2011; 301:G1004G1013. doi: 10.1152/ajpgi.00167.2011.

19. Yeo M, Kim DK, Park HJ, et al. Loss of transgelin in repeated bouts of ulcerative colitis-induced colon carcinogenesis. Proteomics. 2006; 6: 1158-65. doi: 10.1002/pmic.200500390.

20. Gommeaux J, Cano C, Garcia S, Gironella M, et al.Colitis and colitis-associated cancer are exacerbated in mice deficient for tumor protein 53induced nuclear protein 1.Mol Cell Biol. 2007; 27: 2215-28. doi: 10.1128/MCB.01454-06.

21. Okayasu et al. A novel method in the induction of reliable experimental acute and chronic ulcerative colitis in mice. Gastroenterology, 1990; 98 (3): 694702. http://ci.nii.ac.jp/naid/10010110133/en/

22. Bradford MM.A rapid and sensitive method for the quantitation of microgram quantities of protein utilizing the principle of protein-dye binding. Anal Biochem. $\quad$ 1976; 248-54. http://dx.doi.org/10.1016/0003-2697(76)90527-3.

23. Guarner F, Malagelada JR. Gut flora in health and 
disease. Lancet. 2003; 361: 512-519. doi: http://dx.doi.org/10.1016/S0140-6736(03)12489-0.

24. Torres MI, Rios A. Current view of the immunopathogenesis in inflammatory bowel disease and its implications for therapy. World J Gastroenterol. 2008; 14: 1972-1980. doi: 10.3748/wjg.14.1972.

25. Kokesova A, Frolova L, Kverka M, Sokol D, et al.Oral administration of probiotic bacteria (E. coli Nissle, E. coli 083 , Lactobacillus casei) influences the severity of dextran sodium sulfateinduced colitis in BALB/c mice. Folia Microbiol. 2006; 51: 478-484. http://dx.doi.org/10.1007/BF02931595.

26. Chen LL, Wang $\mathrm{XH}$, Cui Y, et al.Therapeutic effects of four strains of probiotics on experimental colitis in mice. World J Gastroenterol. 2009; 15: 321-7. doi: 10.3748/wjg.15.321.

27. Tursi A, Brandimarte G, Papa A, Giglio A, et al.Treatment of relapsing mild-to-moderate ulcerative colitis with the probiotic VSL\#3 as adjunctive to a standard pharmaceutical treatment: a double-blind, randomized, placebo-controlled study. Am J Gastroenterol. 2010; 105: 2218-27. doi: 10.1038/ajg.2010.218.

28. Mencarelli A, Distrutti E, Renga B, et al. Probiotics modulate intestinal expression of nuclear receptor and provide counter-regulatory signals to inflammation-driven adipose tissue activation. PLoS One. 2011; 6: e22978. doi: 10.1371/journal.pone.0022978.

29. Oliveira M, Bosco N, Perruisseau G, et al.Lactobacillus paracasei Reduces Intestinal Inflammation in Adoptive Transfer Mouse Model of Experimental Colitis. ClinDev Immunol. 2011; 807483. doi: 10.1155/2011/807483.

30. Toumi R, Abdelouhab K, Rafa H, Soufli I, RaissiKerboua D, Djeraba Z, et al.Beneficial role of the probiotic mixture Ultrabiotique on maintaining the integrity of intestinal mucosal barrier in DSSinduced experimental colitis.Immunopharmacollmmunotoxicol. 2013;35(3):403-9. doi: 10.3109/08923973.2013.790413.

31. Chapman TM, Plosker GL, Figgitt DP. Spotlight on VSL\#3 probiotic mixture in chronic inflammatory bowel diseases. BioDrugs. 2007; 21: 61-63. http://dx.doi.org/10.2165/00063030-20072101000007

32. Chapman TM, Plosker GL, Figgitt DP. VSL\#3 probiotic mixture: a review of its use in chronic inflammatory bowel diseases. Drugs. 2006; 66:
1371-1387. http://dx.doi.org/10.2165/00003495200666100-00006

33. Wirtz S, Neufert C, Weigmann B, Neurath MF. Chemically induced mouse models of intestinal inflammation. Nat Protoc. 2007; 2: 541-6. doi : 10.1038/nprot.2007.41

34. Dai C, Zheng CQ, Meng FJ, et al.VSL\#3 probiotics exerts the anti-inflammatory activity via PI3k/Akt and NF-kappaB pathway in rat model of DSSinduced colitis. Mol Cell Biochem. 2013; 374:1-11. doi: 10.1007/s11010-012-1488-3.

35. Shen J, Zuo ZX, Mao AP. Effect of probiotics on inducing remission and maintaining therapy in ulcerative colitis, Crohn's disease, and pouchitis: meta-analysis of randomized controlled trials. Inflamm Bowel Dis. 2014; 20: 21-35. doi: 10.1097/01.MIB.0000437495.30052.be.

36. Kullmann F, Messmann H, Alt M, Gross V, Bocker T, et al. Clinical and histopathological features of dextran sulfate sodium induced acute and chronic colitis associated with dysplasia in rats. Int J Colorectal Dis. 2001; 16 (4):238-46. doi 10.1007/s003840100311.

37. Shinoda $M$, Shin-Ya $M$, Naito $Y$, Kishida $T$, Ito $R$, Suzuki $N$, et al.Early-stage blocking of Notch signaling inhibits the depletion of goblet cells in dextran sodium sulfate- induced colitis in mice. J Gastroenterol. 2010; 45: 608-617. doi: 10.1007/s00535-010-0210-z.

38. McCormack G, Moriaty D, O'Donoghue DP, McCormick PA, Sheahan K, Baird AW. Tissue cytokine and chemokine expression in inflammatory bowel disease. Inflamm. Res. 2001; 50 491-495. doi: 10.1007/PL00000223.

39. Madsen K, Cornish A, Soper P, McKaigney C, et al. Probiotic bacteria enhance murine and human intestinal epithelial barrier function. Gastroenterology. 2001; 121: 580-91. doi: http://dx.doi.org/10.1053/gast.2001.27224.

40. Gaudier E, Michel C, Segain JP, Cherbut C, Hoebler C. The VSL\# 3 probiotic mixture modifies microflora but does not heal chronic dextran-sodium sulfateinduced colitis or reinforce the mucus barrier in mice. J. Nutr. 2005; 135 (12) 2753-2761. http://jn.nutrition.org/content/135/12/2753.

41. Camacho-Barquero L, Villegas I, Sánchez-Calvo JM, Talero $E$, et al.Curcumin, a Curcuma longa constituent, actson MAPK p38 pathwaymodulating COX-2 andiNOSexpression in chronic experimental colitis. IntImmunopharmacol. 2007; 7: 333-42. doi: 10.1016/j.intimp.2006.11.006. 
42. Romero $M$, Artigiani $R$, Costa $H$, Oshima $C T$, Miszputen $S$, Franco M.Evaluation of the immunoexpression of COX-1, COX-2 and p53 in Crohn's disease. ArqGastroenterol. 2008; 45: 295$300 . \quad$ http://dx.doi.org/10.1590/S000428032008000400007.

43. Otte JM, Mahjurian-namari R, Marca S, Werner I, Schmidt WE, Schmitz F. Probiotics regulate the expression of COX-2 in intestinal epithelial cells. Cáncer de Nutr. 2009; 61: 103-13. doi: 10.1080/01635580802372625.

44. Esposito E, lacono A, Bianco G, Autore G, et al.Probiotics reduce the inflammatory response induced by a high-fat diet in the liver of young rats. J Nutr. 2009; 139: 905-11. doi: 10.3945/jn.108.101808.

45. Peran L, Camuesco D, Comalada M, Bailon E,et al. A comparative study of the preventative effects exerted by three probiotics, Bifidobacteriumlactis, Lactobacillus casei and Lactobacillus acidophilus, in the TNBS model of rat colitis. J ApplMicrobiol. 2007; 103: 836-844. doi: 10.1111 / j.13652672.2007.03302.x.

Las opiniones que contenga este artículo son de su(s) autor(es) y no necesariamente representan la opinión oficial de la Universidad Simón Bolivar, la Revista Ciencia e Innovación en Salud o de sus autoridades. 